University of Nebraska - Lincoln

DigitalCommons@University of Nebraska - Lincoln

$2-6-2000$

\title{
Exact analytic relation between quantum defects and scattering phases with applications to Green's functions in quantum defect theory
}

\author{
V. E. Chernov \\ Voronezh State University, Voronezh, Russia \\ N. L. Manakov \\ Voronezh State University, manakov@phys.vsu.ru \\ Anthony F. Starace \\ University of Nebraska-Lincoln, astarace1@unl.edu
}

Follow this and additional works at: https://digitalcommons.unl.edu/physicsstarace

Part of the Physics Commons

Chernov, V. E.; Manakov, N. L.; and Starace, Anthony F., "Exact analytic relation between quantum defects and scattering phases with applications to Green's functions in quantum defect theory" (2000). Anthony $F$. Starace Publications. 133.

https://digitalcommons.unl.edu/physicsstarace/133

This Article is brought to you for free and open access by the Research Papers in Physics and Astronomy at DigitalCommons@University of Nebraska - Lincoln. It has been accepted for inclusion in Anthony F. Starace Publications by an authorized administrator of DigitalCommons@University of Nebraska - Lincoln. 


\title{
Exact analytic relation between quantum defects and scattering phases with applications to Green's functions in quantum defect theory
}

\author{
V. E. Chernov ${ }^{1}$, N. L. Manakov ${ }^{1}$, and A. F. Starace ${ }^{2}$ \\ ${ }^{1}$ Department of Physics, Voronezh State University, 394693 Voronezh, Russia \\ 2 Department of Physics and Astronomy, University of Nebraska-Lincoln, Lincoln, Nebraska 68588-0111, USA \\ Email: N. L. Manakov: manakov@thp.vsu.ru ; A. F. Starace: astarace1@unl.edu
}

\begin{abstract}
The relation between the quantum defects, $\mu_{\lambda}$, and scattering phases, $\delta_{\lambda}$, in the single-channel Quantum Defect Theory (QDT) is discussed with an emphasis on their analyticity properties for both integer and noninteger values of the orbital angular momentum parameter $\lambda$. To derive an accurate relation between $\mu_{\lambda}$ and $\delta_{\lambda}$ for asymptotically-Coulomb potentials, the QDT is formally developed for the Whittaker equation in its general form "perturbed" by an additional short-range potential. The derived relations demonstrate that $\mu_{\lambda}$ is a complex function for abovethreshold energies, which is analogous to the fact that $\delta_{\lambda}$ is complex for below-threshold energies. The QDT Green's function, $G_{\lambda}$, of the "perturbed" Whittaker equation is parameterized by the functions $\delta_{\lambda}$ and $\mu_{\lambda}$ for the continuous and discrete spectrum domains respectively, and a number of representations for $G_{\lambda}$ are presented for the general case of noninteger $\lambda$. Our derivations and analyses provide a more general justification of known results for nonrelativistic and relativistic cases involving Coulomb potentials and for a Coulomb plus point dipole potential.
\end{abstract}

Keywords: Calculations and mathematical techniques, atomic and molecular physics, multiphoton ionization, excitation, highly excited states, Rydberg states, Green's functions, quantum defect theory, scattering phases, Whittaker equation

\section{Introduction}

The quantum defect theory (QDT) is one of the most effective semiempirical methods in atomic and molecular physics. Based on the information known from the spectra $\varepsilon_{n}$ of one-electron excitations, this approach yields good estimates for the non-Coulomb part of scattering phases $\delta_{l}(\varepsilon)$ (where $l$ is the orbital angular momentum quantum number), for the bound-bound and bound-free matrix elements (e.g., the oscillator strengths and the photoionization crosssections), and for more complicated single-channel atomic parameters such as polarizabilities, hyperpolarizabilities, and cross-sections of other multiphoton processes determined by higher orders of perturbation theory. At present, the multichannel modifications of QDT are effective tools for the analysis of complex spectra and collision processes, especially in molecular applications. Because the QDT has a long history, we discuss briefly in this introductory section that aspect of this history which is the focus of the present paper: the relationship between quantum defects and phase shifts (referred to henceforward as the $\delta-\mu$ relation) and especially the fact, which is discussed here for the first time, that the quantum defect is a complex-valued function for above-threshold energies. With this context established, we then outline the results of the present paper.

\subsection{Brief review of QDT formulas relating the quantum defect to the phase shift}

After the first application of the Coulomb-like approximation to the systematic analysis of oscillator strengths in nonhydrogenic atoms [1], the next principal steps in this area were made by Seaton [2,3] and Ham [4], who considered the quantum defect as a function of energy, $\mu_{l}(\varepsilon)$, whose values at $\varepsilon=\varepsilon_{n}$ are determined by the difference between the experimental one-electron energies, $\varepsilon_{n}$, and the pure Coulomb ones, i.e.,

$$
\mu_{l}\left(\varepsilon_{n}\right)=n-Z v\left(\varepsilon_{n}\right)
$$

where $Z$ is the residual ion charge, and where $n$ and $v(\varepsilon)=$ $(-2 \varepsilon)^{-1 / 2}$ are the principal and "effective" principal quantum numbers for a series of levels with orbital angular momentum $l$. These authors analyzed some of the general properties of $\mu_{l}(\varepsilon)$ and so they went beyond the Coulomb- 
like approximation to create the QDT. Seaton's famous relation [2],

$$
\cot \delta_{l}=\cot \pi \mu_{l},
$$

between $\mu(\varepsilon)$ and $\delta(\varepsilon)$ in the near-threshold region of the continuum put in place the basis for wide applications of QDT to the analysis of photoionization cross-sections and scattering phases (see, e.g., [5,6]). Somewhat later, Seaton proposed an extended form of this relation [3]

$$
\cot \delta_{l}=(1-\exp (-2 \pi Z / k)) \cot \pi \mu_{l},
$$

which was intended to be applicable in a wider interval of $\varepsilon$ $>0$. Here $k=(2 \varepsilon)^{1 / 2}$. Seaton was interested mainly in determining $\delta_{1}(\varepsilon)$ in terms of $\mu_{l}(\varepsilon)$ extrapolated into the continuum from the bound state region $\varepsilon<0$, so that both $\cot \delta_{1}$ and $\cot \pi \mu_{1}$ in (3) are considered as real functions for $\varepsilon>0$. The equation (3) was later rederived in [8] using effective range theory for near-threshold energies.

A more accurate form of $\delta-\mu$ relation for transition from positive to negative energies $\varepsilon$ was derived for the first time by Norman [9], who pointed to inaccuracies in Seaton's derivation of relation (3). Norman also used effective range theory, but treated the fundamental relations of this theory for positive and negative energies more precisely than in [8]. We present Norman's relation [9] here in a form analogous to (3),

$$
\begin{aligned}
& \cot \delta_{l}(\varepsilon>0) \rightarrow \\
& \quad(1-\exp (\mathrm{i} 2 \pi Z v)) \cot \pi \mu_{l}(\varepsilon<0)-\mathrm{i} \exp (\mathrm{i} 2 \pi Z v),
\end{aligned}
$$

where the arrow indicates the analytic continuation of cot $\delta_{l}$ to energies $\varepsilon \equiv-1 / 2 v^{2}<0$. Considering the relation (4) as an equality with $v=\mathrm{i} / k$ on the right-hand side, we see that the resulting equation differs from (3) by the last term on the right-hand side of (4); this term is purely imaginary for positive $\varepsilon$ (or equivalently for $v=\mathrm{i} / k$ ). Thus, considering (4) as an equality with $\varepsilon>0$ on both sides and writing its real part only, we obtain the following more precise form of equation (3)

$$
\cot \delta_{l}(\varepsilon>0)=(1-\exp (-2 \pi Z / k)) \operatorname{Re}\left[\cot \pi \mu_{l}(\varepsilon>0)\right] \text {. }
$$

Thus Norman's result, although not remarked upon explicitly in [9], shows that the accurate analytical $\delta-\mu$ relation requires that $\delta$ and $\mu$ be considered as complex for negative and positive energies, respectively. Seaton had earlier observed that $\mu(\varepsilon)$ becomes complex for positive energies if results of Ham [4] are employed (see the first footnote on p. 509 of [3]), but he did not pursue the matter. The corresponding complexity of $\delta_{l}(\varepsilon)$ for $\varepsilon<0$ is not surprising, as it follows from the well-known relation of scattering theory that for the discrete spectrum energies one has

$$
\cot \delta_{l}(\varepsilon) \rightarrow \mathrm{i} \text { for } \varepsilon \rightarrow \varepsilon_{n},
$$

which determines the $S$-matrix poles corresponding to the bound states. (Alternatively, (5) follows from the asymptotic boundary condition on discrete state wave functions [10].) Using the definition (1) for the quantum defect, the relation (4) for $\varepsilon=\varepsilon_{n}$ reduces immediately to (5), unlike the relation (3). Note, that for the Coulomb plus short-range potential the substitution (5) for the transition from continuum to bound state energies was used first by Landau and Smorodinskii [10].

The relation (4) is important and necessary for correct analytic manipulations involving the functions $\mu(\varepsilon)$ and $\delta(\varepsilon)$. It permitted the derivation of the QDT Green's function for an optical (valence) atomic electron for the case of integer values of $l$ [11]. This Green's function is convenient for calculating radial dipole matrix elements in high orders of perturbation theory, e.g., for multiphoton calculations. Using an interpolation procedure for the determination of (real) $\mu_{l}(\varepsilon)$ at energies below threshold in the intervals $\varepsilon_{n}<$ $\varepsilon<\varepsilon_{n+1}$, such an approach enables calculations of the multiphoton cross-sections for the simplest atoms with the same precision as the Bates-Damgaard calculations [1] of oscillator strengths (see, e.g., $[12,13])$. An important advantage of the QDT approach as compared to more ab initio theoretical methods is that the resonance structure of the cross-sections coincides with the experimental resonances owing to the use of the experimental data for $\varepsilon_{n}$. Note that the QDT Green's function is also useful in collision problems [14].

A treatment of the QDT in a more general form than previously performed was given in $[15,16]$. In addition to the nonrelativistic Coulomb problem, these authors considered the cases of a free electron and of an electron in a long-range dipole potential. However, these authors were interested mainly in the extraction of a set of convenient parameters (" the QDT parameters") describing the core effects on bound and continuum state wavefunctions without analyzing the $\delta-\mu$, relation. Moreover they “ ... dropped Seaton's distinction between $\delta$ and $\pi \mu$ for $\varepsilon>0$ " (see p. 1498 of [15]). The most detailed results on the general form of QDT were derived in [16] based on a special prescription for the definition of the Jost functions for the case of singular potentials. That work deals with six QDT parameters as real functions of energy and angular momentum. Later, some relationships between these parameters were given in [17]. Furthermore, in order to extend the Jost function formalism to the case of singular potentials, reference [16] employed different representations for the QDT basis functions above and below threshold obtained using a nonstandard asymptotic form of the confluent hypergeometric function (in Appendix $\mathrm{C}$ we present further details on this matter and on the relation to the present work).

Concerning the QDT Green's function, this was constructed in [15] based on the regular and irregular solutions of the Schrödinger equation. But it seems that the authors did not intend to employ these Green's functions for the calculation of high-order matrix elements or for the crosssections of multiphoton processes and so they worked only with so-called regular (or "smooth") Green's functions (introduced by Fano [18]), which do not contain any poles and are useful in multichannel applications, especially to Rydberg atoms and molecules. (For details of such applications see, e.g., [19-22].) Moreover, the bound and continuum eigenfunctions were obtained without using the Green's func- 
tions, through a direct analysis of the radial Schrödinger equation solutions for bound and continuum states; hence all results of $[15,16]$ are independent of the explicit $\delta-\mu$ relation. Furthermore, for those problems considered in [15] that involve the radial Schrödinger equation with a noninteger orbital parameter $l$, a relationship between $\mu$ and $\delta$ has yet to be derived, since the Norman relation (4) is valid for integer l only.

The relativistic generalization of QDT for a single electron described by the Dirac equation was addressed in [23,24]. Zilitis [23] confirmed that the relation (2) applies in the relativistic case at threshold. He noted that Seaton had derived a more general expression (3), but commented that he was "inclined ... [to] agree with Norman [9] that this more general relationship is not correct." Johnson and Cheng [24], however, aimed to generalize Seaton's more general equation (3) to the relativistic case, based on [2-4]. In equation (34) of [24] an analytic function $\mathcal{B}$ is defined which is real below threshold and complex above the threshold. However, in analytically continuing a function $\beta(\varepsilon)$ (see Eq. (37) of [24]) above threshold, only the real part of $\mathcal{B}$ is kept, which is evidently non-analytic. Consequently, Johnson and Cheng [24] obtained the following $\delta-$ $\mu$ relation:

$$
\begin{aligned}
& \cot \delta_{\lambda}(\bar{\varepsilon})= \\
& \quad\left[1-\cos (2 \pi \lambda) \mathrm{e}^{\mathrm{i} 2 \pi Z \bar{v}}\right] \cot \pi \mu_{\lambda}(\bar{\varepsilon})-\sin (2 \pi \lambda) \mathrm{e}^{\mathrm{i} 2 \pi Z \bar{v}} .
\end{aligned}
$$

Here $\bar{\varepsilon}$ is equal to the total energy of the relativistic electron, $E$, in units of its rest mass, $m c^{2}, \lambda$ is a relativistic parameter (defined explicitly in Appendix B below) which in the non-relativistic limit becomes the orbital angular momentum $l$ of the electron, and $\bar{v}$ is an energy-dependent parameter defined as

$$
\bar{v} \equiv \alpha \bar{\varepsilon} /\left(1-\bar{\varepsilon}^{2}\right)^{1 / 2},
$$

which in the non-relativistic limit takes the usual form for the effective principal quantum number $(c f .(1)), 1 /(-2 \varepsilon)^{1 / 2}$. One readily sees that in the non-relativistic limit, equation (6) agrees with the more general equation (3) of Seaton but not with the correct equation (4) of Norman. In particular, (6) does not satisfy (5) at the discrete state energies.

The relativistic case was revisited by Zilitis [25] and by Goldberg and Pratt [26]. However, Zilitis simply notes the results of Johnson and Cheng [24] without discussion, while Goldberg and Pratt reproduce the results of [24]. QDT analysis for the Dirac equation was also given by Chang [27], based on the ideas of the generalized QDT $[15,16]$, without mentioning the $\delta-\mu$ relation. The correct form of this relation in the relativistic case was stated without discussion or detailed derivation in [28]. This relation corresponds to (6), and coincides with (4) in the non-relativistic case and yields equation (5) for bound states. It was used in [28] for the derivation of the one-electron Green's function in relativistic QDT by a method similar to that employed in the nonrelativistic case (see Ref. [11]). Using an analogous correct relationship between the quantum defect and the phase shift, the QDT Green's function was pre- sented recently for the case of Rydberg electron states in polar molecules in reference [29], which refers to the results presented in [28].

\subsection{Outline of the present paper}

As the above survey shows, although the $\delta-\mu$ relation is one of the fundamental results of QDT, it requires some additional investigation. Many key references in the field of QDT present a $\delta-\mu$ relation which is not quite correct. Furthermore, those references which present a correct analytic relation have done so for only specific cases and in a number of instances have merely stated the result without derivation or discussion. Specifically, the correct non-relativistic result (4), for example, was derived in [9] based on methods of effective range theory for a repulsive Coulomb potential with further examination of the attractive potential case. Furthermore, since the effective range approach is justified only in the near-threshold energy region, an additional independent analysis of the relation (4) is desirable. Secondly, an accurate generalization of that result to the general case of noninteger orbital parameters is needed (as is required, e.g., in the Coulomb plus dipole field case). Finally, a detailed derivation of the correct relation corresponding to (6) for the relativistic case has yet to be presented, although the correct result was stated in [28].

In Section 2 we analyze all of these problems for the general form of the Whittaker equation perturbed by a shortrange potential with a radius $r_{c}$. Using QDT ideas, the treatment is based on knowledge of the known spectrum of the corresponding Sturm-Liouville problem for the perturbed Whittaker equation on the interval $0 \leq r<\infty$. All the known $\delta-\mu$ relations are special cases of our key relations given in (32) below for the general case of non-integer $\lambda$. For nearthreshold energies $\epsilon>0$ and $\lambda=l$ these relations give an improved form (38) of Seaton's formula (3).

Based on the $\delta-\mu$ relation given in Section 2, in Section 3 we construct the Green's function of the general Whittaker equation for the $r, r^{\prime}>r_{\mathrm{c}}$ domain. It contains all the information needed for obtaining the correctly normalized QDT wavefunctions for bound and continuum states in a most economical way. As has been mentioned above, such a general Green's function is useful, in particular, for calculations of cross-sections for multiphoton processes using the QDT approach [13]. The results presented are applicable to all physical problems for which the radial Schrödinger equation reduces to the general form of the Whittaker (or confluent hypergeometric) equation. Finally, in appendices we present some further results. In Appendix A the $\delta-\mu$ relation is derived in terms of an alternate set of QDT basis functions. In Appendix B we specify the parameters of the general Whittaker equation for the three most important physical applications of QDT: (i) a nonrelativistic or (ii) a relativistic electron in a Coulomb potential, and (iii) an electron in the Coulomb plus point dipole potential. Thus, our general development provides an accurate and unified derivation and additional justification of some known 
QDT-results for these important physical problems. Lastly, Appendix C provides a more detailed discussion of the relation of this paper to references [15-18].

Before proceeding with the program outlined above, we make some observations on the approach we follow here, namely, that of considering the quantum defect, $\mu(\varepsilon)$, and the phase shift, $\delta(\varepsilon)$, as complex functions of $\varepsilon$. This approach may seem strange to some readers. We hasten to add that in the regions where one expects these functions to be real, i.e., $\mu$ for energies below threshold, and $\delta$ for energies above threshold, they are. Our purpose here is simply to present the correct analytic $\delta-\mu$ relation for the same reasons stated long ago by Morse and Feshbach [30] for the study of complex functions, namely that extending the study of $\mu$ and $\delta$ to complex values is done " ... for reasons of completeness and convenience [as well as for] ... the insight we shall obtain into the general properties of functions." Pragmatic readers may also ask what is the practical effect of considering $\mu(\varepsilon)$ and $\delta(\varepsilon)$ as complex functions. Although the exact relation between $\delta$ and $\mu$ allows us to present for $\varepsilon>0$ and $\lambda=l$ an improved form of Seaton's formula (3), we do not expect a great improvement in extrapolation procedures for quantum defects or phase shifts (cf. Table 1). Nevertheless, this small practical importance for analysis of spectroscopic data should not detract from the significance of the exact relation for theoretical analyses. Indeed, as shown in this paper, using the proper relations in equation (32) for analytically continuing $\mu(\varepsilon)$ and $\delta(\varepsilon)$ above or below threshold will have the greatest application in theoretical analyses. As shown in this paper, for example, it permits one to obtain an analytical representation for the appropriate Green's function (given in Equation (43) below), which has a single form for energies both above and below threshold and is valid for the case of integer as well noninteger values of the orbital angular momentum parameter $\lambda$.

\section{Relation of $\delta$ and $\mu$ in the QDT analysis of the Whittaker equation}

\subsection{Background results for the Whittaker equation}

We consider the Whittaker-like equation with an additional short-range potential $u(z)$

$$
\begin{aligned}
\hat{L}_{u}[f] \equiv\left\{\frac{\mathrm{d}^{2}}{\mathrm{~d} z^{2}}-\frac{1}{4}+\frac{Z \nu}{z}+\frac{1 / 4-(\lambda+1 / 2)^{2}}{z^{2}}\right. \\
+u(z)\} f(z)=0 .
\end{aligned}
$$

Here $\lambda$ is supposed to have a fixed (real) value and $v$ is considered to be a complex parameter. $Z>0$ is a constant depending upon the particular physical application (e.g., the nuclear charge). The real radial variable $r$ is related to $z$ as follows: $z=2 r / v$, where $0 \leq r<\infty$. The short-range potential $u(z)$ satisfies the following conditions: $u(z) \sim \mathrm{O}\left(r^{-1}\right)$ for $r \rightarrow \mathrm{o}$, and $u(z)=\mathrm{o}$ for $r \geq r_{\mathrm{c}}$. The explicit form of $u$ is un- known, but we suppose that adding this short-range potential does not change the characteristic features of the corresponding "unperturbed" Sturm-Liouville problem, $\hat{L}_{0}[f]$ $=0$. Namely, we suppose that the continuous spectrum of (7) corresponds to imaginary values of the spectral parameter $v$,

$$
v=\mathrm{i} / k, \quad k>0 ;
$$

and that the discrete spectrum corresponds to the following eigenvalues of the parameter $v$ :

$$
\begin{gathered}
v=v_{n \lambda}, \quad n=0,1,2, \ldots ; \\
v_{n \lambda} \rightarrow+\infty \quad \text { as } n \rightarrow \infty .
\end{gathered}
$$

Note that in physical applications $n$ is an analogue of the radial quantum number $n_{\mathrm{r}}$. Moreover, for the pure Coulomb potential $(u=0)$

$$
\nu_{n \lambda}^{0}=\frac{1}{Z}(n+\lambda+1)
$$

Let us first present well-known results for the solutions of (7) with $u \equiv 0$, i.e., the results for the Coulomb problem with noninteger $l=\lambda$ which are necessary for our further considerations. In this case, equation (7) becomes the standard Whittaker equation, which has the following widelyused pair of solutions (see, e.g., [31,32]),

$$
M_{Z v, \lambda+1 / 2}(z) \text { and } W_{Z v, \lambda+1 / 2}(z)
$$

which are linearly independent for all $\lambda$. For the purposes of this paper we introduce the following modified Whittaker functions:

$$
\begin{aligned}
\mathcal{M}^{+}(z) & =\frac{M_{Z \nu, \lambda+\frac{1}{2}}(z)}{\Gamma(2 \lambda+2)} \\
& =\mathrm{e}^{\mathrm{i} \pi Z \nu}\left(\mathcal{W}^{-}(z)-\mathrm{e}^{-\mathrm{i} \pi \lambda} \mathcal{W}^{+}(z)\right), \\
\mathcal{M}^{-}(z) & =\frac{M_{Z \nu,-\lambda-\frac{1}{2}}(z)}{\Gamma(-2 \lambda)} \\
\mathcal{W}^{ \pm}(z) & =\frac{W_{ \pm Z \nu, \lambda+\frac{1}{2}}( \pm z)}{\Gamma(1+\lambda \pm Z \nu)}
\end{aligned}
$$

where we assume $-z=\mathrm{e}^{\mathrm{i} \pi} z$ and $\operatorname{Im} z \leq^{\wedge} 0$.

Above the threshold, i.e., for $v=i / k$, it is convenient to use another base pair: $\mathfrak{f}_{\lambda}(k, r)$ and $\mathfrak{g}_{\lambda}(k, r)$. We define them by

$$
\begin{aligned}
\mathcal{M}^{+} \leftrightarrow \frac{2 \mathrm{e}^{-\mathrm{i} \pi(1+\lambda) / 2} \mathrm{e}^{-\frac{\pi Z}{2 k}}}{\left|\Gamma\left(1+\lambda+\frac{\mathrm{i} Z}{k}\right)\right|} \mathfrak{f}_{\lambda}(k, r), \\
\mathcal{W}^{ \pm} \leftrightarrow \frac{\mathrm{e}^{\mp \mathrm{i} \pi(1+\lambda) / 2} \mathrm{e}^{\frac{\pi Z}{2 k}}}{\left|\Gamma\left(1+\lambda+\frac{\mathrm{i} Z}{k}\right)\right|}\left(\mathfrak{f}_{\lambda}(k, r) \pm \mathrm{ig}_{\lambda}(k, r)\right),
\end{aligned}
$$

where $\longleftrightarrow$ means the substitution $v \longleftrightarrow \mathrm{i} / k$. Using the well-known asymptotic expansions for Whittaker functions $[31,32]$,

$$
\begin{aligned}
W_{Z \nu, \lambda+\frac{1}{2}}(z) & \sim z^{Z \nu} \mathrm{e}^{-z / 2}, \\
W_{-Z \nu, \lambda+\frac{1}{2}}(-z) & \sim(-z)^{-Z \nu} \mathrm{e}^{z / 2},
\end{aligned}
$$


one can obtain the following asymptotic forms for $\mathfrak{f}_{\lambda}, \mathfrak{g}_{\lambda}$ at $r \rightarrow \infty$ :

$$
\mathfrak{f}_{\lambda}(k, r) \sim \sin \Delta_{\lambda}(r) \quad \mathfrak{g}_{\lambda}(k, r) \sim \cos \Delta_{\lambda}(r),
$$

where $\Delta_{\lambda}(r)=k r+(Z / k) \ln 2 k r-\pi \lambda / 2+\sigma_{\lambda}$, and where $\sigma_{\lambda}=$ $\arg \Gamma(1+\lambda-\mathrm{i} Z / k)$.

Rather than the $\left(\mathcal{M}^{+}, \mathcal{W}^{+}\right)$and $\mathcal{W}^{ \pm}$bases, another pair of linearly independent solutions is used in QDT. This pair is denoted (to within a constant factor) as $y_{1,2}$ in [7], as $y(\kappa$, $\pm \lambda, z)$ in [6], and as $\boldsymbol{y}_{\mathrm{R}, \mathrm{I}}$ in [24]. In the present paper these solutions are denoted as $f_{\lambda}(z)$ and $f_{-\lambda-1}(z)$. Here we express them in terms of the $\left(\mathcal{M}^{+}, \mathcal{W}^{+}\right), \mathcal{W}^{ \pm}$, and $\left(\mathfrak{f}_{\lambda}, \mathfrak{g}_{\lambda}\right)$ base pairs as:

$$
\begin{aligned}
\mathrm{f}_{\lambda}= & \nu^{\lambda+1} \mathcal{M}^{+} \\
\mathrm{f}_{-\lambda-1}= & \nu^{-\lambda} \mathcal{M}^{-} \\
= & -\nu^{\lambda+1} \mathcal{A}(\nu, \lambda) \frac{\mathcal{W}^{+} \sin 2 \pi \lambda+\mathcal{M}^{+} \sin \pi(\lambda+Z \nu)}{\sin \pi(Z \nu-\lambda)} \\
= & \nu^{\lambda+1} \mathcal{A}(\nu, \lambda) \mathrm{e}^{\mathrm{i} \pi Z \nu} \\
& \times\left[\mathrm{e}^{\mathrm{i} \pi \lambda} \mathcal{W}^{+}-\frac{\sin \pi(Z \nu+\lambda)}{\sin \pi(Z \nu-\lambda)} \mathcal{W}^{-}\right] \\
\leftrightarrow & 2 \mathrm{i} k^{\lambda} \frac{\mathrm{e}^{-\mathrm{i} \pi \lambda-\mathrm{i} \sigma_{\lambda}} \mathrm{e}^{-\frac{\pi Z}{2 k}}}{\Gamma\left(-\lambda+\mathrm{i} \frac{Z}{k}\right)} \\
& \times \frac{\mathfrak{f}_{\lambda}\left(\mathrm{e}^{-\frac{2 \pi Z}{k}}-\cos 2 \pi \lambda\right)-\mathfrak{g}_{\lambda} \sin 2 \pi \lambda}{\mathrm{e}^{-2 \pi \mathrm{i} \lambda-\frac{2 \pi Z}{k}}-1}
\end{aligned}
$$

Following Seaton's notation, we introduce here the factor

$$
\mathcal{A}(\nu, \lambda)=\frac{\Gamma(1+\lambda+Z \nu)}{\nu^{2 \lambda+1} \Gamma(Z \nu-\lambda)} .
$$

Note that $f_{\lambda}$ and $f_{-\lambda-1}$ are energy independent at small $r$ :

$$
\mathrm{f}_{\lambda}(z \rightarrow 0) \rightarrow \frac{(2 r)^{\lambda+1}}{\Gamma(2 \lambda+2)}
$$

The difference between the basis pairs (11) and $\left(f_{\lambda}, f_{-\lambda-1}\right)$ is that, for non-integer $2 \lambda$, the latter basis functions are analytic in $v$ about some neighborhood of infinity (or, equivalently, the functions $\mathrm{f}_{\lambda}$ and $\mathrm{f}_{-\lambda-1}$ are analytic in $v^{-1}$ near zero). For $2 \lambda=2 l$ is integer, $f_{-\lambda-1}$ can be defined in the limit $\lambda \rightarrow l$, but the resulting function is non-analytic in $v$. The appropriate base pair in this case is analyzed in $[3,4]$. We present its explicit form in Appendix A.

\subsection{Real values of the $v$ parameter: the quantum defect as a function of $v$}

Returning to equation (7), let us consider its regular solution (at $z \rightarrow 0), f_{\lambda}^{\mathrm{reg}}(v ; z)$, which is an entire analytic function in the $v$-plane except for $v=0$. The existence of such a solution is proved by the general theory of differential equations and was discussed in [4]. This solution is not known in the core domain $\left(r<r_{\mathrm{c}}\right)$, but for $r>r_{\mathrm{c}}$ it is a superposi- tion of the linearly independent, entire functions $f_{\lambda}$ and $f$ $\lambda_{-1}$. Note that it is assumed that $2 \lambda$ is non-integer; otherwise we would have to deal with another analytic solution instead of $f_{-\lambda-1}$. We have not analyzed these solutions for integer $2 \lambda$ here, because our final results (for the $\delta-\mu$ relation and for the Green's function) will be applicable for integer $2 \lambda$ as well.

For $r>r_{\mathrm{c}}$, we therefore write the required solution as

$$
f_{\lambda}^{\text {reg }}(v ; z)=f_{\lambda}(z)-\beta_{\lambda-\lambda-1}(z) .
$$

It contains the unknown function $\beta_{\lambda}(v)$, which must be an entire analytic function for all $v \neq 0$, since the functions $\mathrm{f}_{\lambda}$ and $f_{-\lambda-1}$ are analytic. We analyze the function $\beta_{\lambda}$ using arguments similar to those in $[4,24]$. The eigenfunction corresponding to the discrete eigenvalues (9) must satisfy the boundary condition,

$$
f_{\lambda}^{\text {reg }}\left(v=v_{n \lambda} ; z \rightarrow+\infty\right) \rightarrow 0 .
$$

From equations $(12,16,19)$ it can be easily seen that the solution (21) contains both exponentially decreasing and exponentially increasing terms. Since the condition (22) requires the latter to vanish at $v=v_{n \lambda}$, we can determine the coefficient $\beta_{\lambda}$ for the discrete spectrum points:

$$
\begin{aligned}
\beta_{\lambda}^{-1}\left(\nu_{n \lambda}\right) & =\nu_{n \lambda}^{-2 \lambda-1} \frac{\Gamma\left(1+\lambda-Z \nu_{n \lambda}\right)}{\Gamma\left(-\lambda-Z \nu_{n \lambda}\right)} \\
& =\mathcal{A}\left(\nu_{n \lambda}, \lambda\right) \frac{\sin \pi\left(\lambda+Z \nu_{n \lambda}\right)}{\sin \pi\left(\lambda-Z \nu_{n \lambda}\right)},
\end{aligned}
$$

where we have used the well-known reflection formula [34] for the $\Gamma$ function to obtain the last term in (23).

We introduce now the quantum defect function $\mu_{\lambda}(v)$ defined by

$$
\begin{aligned}
\mu_{\lambda}\left(v_{n \lambda}\right) \equiv \mu_{n \lambda} & =Z\left(v_{n \lambda}^{0}-v_{n \lambda}\right) \\
& =n+\lambda+1-Z v_{n \lambda}
\end{aligned}
$$

for the discrete spectrum points given by equation (9). Taking into account (24), we can remove $Z v_{n \lambda}$ from the arguments of the sines in equation (23), so that:

$$
\beta_{\lambda}^{-1}\left(v_{n \lambda}\right)=\mathcal{A}\left(v_{n \lambda}, \lambda\right)\left(\cot \pi \mu_{n \lambda} \sin 2 \pi \lambda-\cos 2 \pi \lambda\right) .
$$

Since $\beta_{\lambda}(v)$ is analytic and $\mu_{\lambda}(v)$ is defined on a subset $v_{n \lambda}$ with an accumulation point ( $c f$. Equation (9)), we may consider that equation (25) defines an analytic function $\beta_{\lambda}(v)$ for all $v \neq 0$. In this case the analytic function $\beta_{\lambda}(v)$ is parameterized with the help of another function, $\cot \pi \mu_{\lambda}(v)$. The function $\beta_{\lambda}(v)$ has no branch point at infinity since $\mathcal{A}$ occurring in (25) has the following asymptotic value for $|v|$ $\rightarrow \infty(|\arg v|<\pi)$ :

$$
Z^{-2 \lambda-1} \mathcal{A}(\nu, \lambda) \sim 1-\frac{\lambda(1+\lambda)(1+2 \lambda)}{6(Z \nu)^{2}}+O\left(\nu^{-4}\right),
$$

which follows from asymptotic expansions of $\Gamma$ functions [34]. Obviously the threshold value $\beta_{\lambda}(+\infty)$ is connected in a simple way with $\cot \pi \mu_{\lambda}(+\infty)$. 
Substituting (25) with $v_{n \lambda}=v$ and $\mu_{n \lambda}=\mu_{\lambda}(v)$ into (21) (and using some properties of the Whittaker functions [31]), we re-write the latter in terms of $\mu_{\lambda}(v)$ and the $\left(\mathcal{M}^{+}\right.$, $\mathcal{W}^{+}$) and $\mathcal{W}^{ \pm}$base pairs:

$$
\begin{aligned}
& f_{\lambda}^{\mathrm{reg}}(\nu ; z)=\frac{\nu^{\lambda+1} \sin 2 \pi \lambda}{\sin \pi\left(2 \lambda-\mu_{\lambda}\right)} \\
& \times \frac{\mathcal{M}^{+}(z) \sin \pi\left(\mu_{\lambda}+Z \nu-\lambda\right)+\sin \pi \mu_{\lambda} \mathcal{W}^{+}(z)}{\sin \pi(Z \nu-\lambda)} \\
&= \frac{\nu^{\lambda+1} \sin 2 \pi \lambda}{\sin \pi\left(2 \lambda-\mu_{\lambda}\right)}\left[\mathrm{e}^{\mathrm{i} \pi Z \nu} \mathcal{W}^{-}(z) \frac{\sin \pi\left(\mu_{\lambda}+Z \nu-\lambda\right)}{\sin \pi(Z \nu-\lambda)}\right. \\
&\left.-\mathrm{e}^{\mathrm{i} \pi\left(\mu_{\lambda}+Z \nu-\lambda\right)} \mathcal{W}^{+}(z)\right] \cdot
\end{aligned}
$$

Note that the first of these identities explicitly involves only real functions for real values of $v$.

\subsection{Imaginary values of the $v$ parameter: the scattering phase as a function of $v$}

For the continuous spectrum (see (8)) another parameterization of the function $\beta_{\lambda}(v=\mathrm{i} / k)$ is preferable. It is a well-known fact that the influence of the short-range core potential results in an additional phase shift, $\delta_{\lambda}$, of the scattering phases in the continuum eigenfunctions, whose asymptotic form becomes (cf. (17))

$$
f_{\lambda}^{\mathrm{reg}}(v=\mathrm{i} / k ; z \rightarrow-\mathrm{i} \infty) \propto \sin \left(\Delta_{\lambda}(r)+\delta_{\lambda}(v)\right) .
$$

For this reason, it is convenient to express $\beta_{\lambda}(v)$ in terms of the phase $\delta_{\lambda}(v)$. To perform such a parameterization, one can use equations $(12,16,19)$ to obtain the asymptotic behavior of the functions $\mathrm{f}_{\lambda}$ and $\mathrm{f}_{-\lambda-1}$ for $z \rightarrow-i \infty$. Substituting these asymptotics into equation (21) and comparing the result with (28) we obtain $\beta_{\lambda}(v)$ in terms of the phase $\delta_{\lambda}(v)$ :

$$
\begin{aligned}
& \beta_{\lambda}^{-1}(\nu=\mathrm{i} / k)=\mathcal{A}(\mathrm{i} / k, \lambda) \\
& \quad \times \frac{1-\exp \left(-\frac{2 \pi Z}{k}-\mathrm{i} \pi \lambda\right)}{\cot \delta_{\lambda}(\mathrm{i} / k) \sin 2 \pi \lambda-\cos 2 \pi \lambda+\exp (-2 \pi Z / k)} .
\end{aligned}
$$

This relation requires some explanation. Indeed, (29) was derived above for $\operatorname{Re} v=0, \operatorname{Im} v>0$. However, $\beta_{\lambda}(v)$ is analytic everywhere in the $v$-plane except at the point $v=0$; the right-hand side of (29) can be considered as an analytical continuation of $\beta_{\lambda}(v)$ for other $v$ in terms of the function cot $\delta_{\lambda}(v)$. But in spite of the analyticity of $\beta_{\lambda}(v)$, the right-hand side of equation (29) does not have an explicitly analytic form at the threshold $|v| \rightarrow \infty$ since it contains the exponents $\exp (2 \pi \mathrm{i} Z v)$, which have an essential singularity at infinity. So for the analyticity of $\beta$ at $v=\infty$ these singularities should be compensated by the essential singularities of cot $\delta_{\lambda}(v)$ near the threshold. It is known [35] that for noninteger $\lambda$ and singular potentials, the $S_{\lambda}(k)$-matrix has an essential singularity at $k=O$ instead of a branch point, as for the case of smooth potentials. But no detailed analysis of this problem similar to the effective range theory approach for the Coulomb potential and integer $\lambda$ is known to us. Thus, perhaps (29) may be considered as a starting point for such analysis. Obviously, the threshold value of $\beta(v)$ is unique and we can obtain it as the limiting case of (29) for $v \rightarrow+\mathrm{i} \infty$ along the positive imaginary semiaxis (taking into account the expansion (26)):

$$
\beta_{\lambda}^{-1}(+\infty)=Z^{2 \lambda+1}\left(\cot \delta_{\lambda}(+\infty) \sin 2 \pi \lambda-\cos 2 \pi \lambda\right) .
$$

Continuing (29) to below-threshold $v$ values, we can consider it for the discrete spectrum points (9). Since the scattering matrix $S_{\lambda}(v)$ has poles at these points, we put $\cot \delta_{\lambda}$ $=\mathrm{i}(c f$. (5)) whereby (29) reduces immediately to the last identity in (23) containing the ratio of sines. Thus, using the parameterization (24), equation (25) can be obtained using only the regular solution (21) for the continuum states. Using equations $(15,20,29)$, this solution can be written as

$$
\begin{aligned}
f_{\lambda}^{\mathrm{reg}}(\nu & =\mathrm{i} / k ; z)=\frac{2 k^{-\lambda-1} \mathrm{e}^{-\frac{\pi Z}{2 k}}}{\left|\Gamma\left(1+\lambda+\mathrm{i} \frac{Z}{k}\right)\right|} \\
& \times \frac{\mathfrak{f}_{\lambda}(k, r) \cot \delta_{\lambda}+\mathfrak{g}_{\lambda}(k, r)}{\cot \delta_{\lambda}-\cot 2 \pi \lambda+\operatorname{cosec} 2 \pi \lambda \exp (-2 \pi Z / k)} .
\end{aligned}
$$

Similarly to the first equality in (27), here we see an explicitly real form of $f_{\lambda}^{\text {reg }}$ for imaginary $v$.

\subsection{Relation between $\delta_{\lambda}$ and $\mu_{\lambda}$}

The two relations (25) (with $v_{n \lambda} \rightarrow v, \mu_{n \lambda} \rightarrow \mu_{\lambda}(v)$ ) and (29) each determine the same analytic function $\beta_{\lambda}(v)$ in the $v$ plane. Apart from unknown $\delta_{\lambda}$ and $\mu_{\lambda}$ parameters, these relations contain simple analytical functions and therefore these formulas can be considered as a relation between quantum defects $\mu_{\lambda}$ and scattering phases $\delta_{\lambda}$. In particular, this relation should allow us:

(i) to express the phases in terms of the quantum defects for real $v$ (i.e., where the phases are not defined from a physical point of view);

(ii) to express the quantum defect in terms of the phases for imaginary $v$ (i.e., where the quantum defect has no physical meaning).

Comparing (25) and (29) yields the following equivalence relations:

$$
\begin{aligned}
& \frac{\cot \delta_{\lambda}(\nu) \sin 2 \pi \lambda-\cos 2 \pi \lambda+\exp (\mathrm{i} 2 \pi Z \nu)}{1-\exp [\mathrm{i} 2 \pi(Z \nu-\lambda)]} \leftrightarrow \\
& \cot \pi \mu_{\lambda}(\nu) \sin 2 \pi \lambda-\cos 2 \pi \lambda .
\end{aligned}
$$

Finally, from this equivalence, we obtain the following fundamental relations between $\delta_{\lambda}$ and $\mu_{\lambda}$ :

$$
\begin{aligned}
& \cot \delta_{\lambda}(\nu) \longrightarrow\left[1-\mathrm{e}^{\mathrm{i} 2 \pi(Z \nu-\lambda)}\right] \cot \pi \mu_{\lambda}(\nu)-\mathrm{ie} \mathrm{e}^{\mathrm{i} 2 \pi(Z \nu-\lambda)} \\
& \cot \pi \mu_{\lambda}(\nu) \longrightarrow \frac{\cot \delta_{\lambda}(\nu)+\mathrm{i} \mathrm{e}^{\mathrm{i} 2 \pi(Z \nu-\lambda)}}{1-\mathrm{e}^{\mathrm{i} 2 \pi(Z \nu-\lambda)}}
\end{aligned}
$$$$
\text { for imaginary } v \text {. }
$$ 
Table 1. Phase shift $\delta\left(k^{2}\right)$ predicted by $\delta-\mu$ relations for ${ }^{3} \mathrm{~S}$ electron- $\mathrm{He}^{+}(1 s)$ scattering.

\begin{tabular}{|c|c|c|c|c|}
\hline \multirow[b]{2}{*}{$1 / 2 k^{2}$ (a.u.) } & \multicolumn{2}{|c|}{ Seaton results } & \multicolumn{2}{|c|}{ Present results } \\
\hline & $\pi \mu\left(k^{2}\right)^{\mathrm{a}}$ & Eq. (3) & Eq. (38) & Eqs. $(35,36)$ \\
\hline 0.9155 & 0.7010 & 0.7057709 & 0.7057901 & 0.7057903 \\
\hline
\end{tabular}

${ }^{\mathrm{a}}$ M. J. Seaton, reference [36].

These relations allow one to reconstruct the scattering phases beyond the imaginary $v$ domain where they are denned physically. Such reconstruction is possible if the quantum defect is known in the real $v$ domain. As noted in the Introduction, the scattering phases become complex if continued into this domain. And vice versa, the function $\mu_{\lambda}(v)$ becomes complex for imaginary $v$. For the discrete spectrum points $v \rightarrow v_{n \lambda}$ the first of relations (32) (taking into account (24)) satisfies the required relation (5).

Although $f_{\lambda}(z)$ and $f_{-\lambda-1}(z)$ are not linearly independent for integer $\lambda$, the relations (32) have no peculiarities for integer values $\lambda=l$ and thus they can be used for integer values of $\lambda$ also, as follows from continuity arguments. Substituting $\lambda=l$ into (32) yields the result (4), which is obtained here without any restrictions on $v$ values. For $v$ values close to the threshold, i.e., for iv $\rightarrow-\infty$, the second of the relations (32) yields the near-threshold Seaton's formula (2). Note, that this result is also obtained immediately upon comparing (30) with the threshold limit of (25).

The $\delta-\mu$ relation (32) allows us to express all relevant functions in a unified form for both above- and belowthreshold energies: e.g., the expressions $(31,27)$ turn into each other under the substitution $v \longleftrightarrow \mathrm{i} / k$ if $\delta$ and $\mu$ are connected by the relationship (32). Because of the importance of this key QDT relationship, we provide in the Appendix $\mathrm{A}$ an alternative (less detailed) derivation which uses base functions other than $f_{\lambda}$ and $f_{-\lambda-1}$.

\subsection{Extended Seaton relation between $\delta_{l}$ and $\mu_{l}$}

If we interpret the first relation in equation (32) as an equality for $v=\mathrm{i} / k$, we have

$$
\cot \delta_{\lambda}=\left[1-\mathrm{e}^{\mathrm{i} 2 \pi(\mathrm{i} Z / k-\lambda)}\right] \cot \pi \mu_{\lambda}-\mathrm{ie}^{\mathrm{i} 2 \pi(\mathrm{i} Z / k-\lambda)} .
$$

where since $v$ is imaginary, $\mu_{\lambda}$ is complex. We write therefore $\mu_{\lambda}$ in terms of its real and imaginary parts,

$$
\mu_{\lambda} \equiv \mu_{r}+\mathrm{i} \mu_{\mathrm{i}}
$$

where in what follows we assume $\lambda=l$ is an integer and where we have omitted (for simplicity) the label $l$ on the rhs of (34). Substituting (34) into (33) and taking the real and imaginary parts of (33) as two real equations, we obtain after some simple algebra:

$$
\begin{aligned}
\cot \delta & =\frac{\left[1-\mathrm{e}^{-2 \pi Z / k}\right] \cot \pi \mu_{\mathrm{r}}}{1+\left(\sinh \pi \mu_{\mathrm{i}} / \sin \pi \mu_{\mathrm{r}}\right)^{2}}, \\
\tanh \pi \mu_{\mathrm{i}} & =\frac{\left(\tanh \pi \mu_{\mathrm{i}}\right)^{2}+\left(\sin \pi \mu_{\mathrm{r}} / \cosh \pi \mu_{\mathrm{i}}\right)^{2}}{1-\mathrm{e}^{2 \pi Z / k}} .
\end{aligned}
$$

Equations $(35,36)$ allow one to obtain an improved formula for the relation of $\delta$ and $\mu$, where now we consider that $\mu$ has both real and imaginary parts, $\mu_{\mathrm{r}}$ and $\mu_{\mathrm{i}}$. For the discrete spectrum, $\mu \equiv \mu_{\mathrm{r}}$ and one may assume that an extrapolation across the $\epsilon=0$ threshold will be smooth. At $\epsilon=0$, $\mu_{\mathrm{i}}=0$. Thus one may also assume that $\mu_{\mathrm{i}}$ will be small for small $k$. We may thus use (36) to obtain for small $\mu_{\mathrm{i}}$,

$$
\tanh \pi \mu_{\mathrm{i}} \approx \sinh \pi \mu_{\mathrm{i}} \approx \frac{\left(\sin \pi \mu_{\mathrm{r}}\right)^{2}}{1-\mathrm{e}^{2 \pi Z / k}} \approx-\left(\sin \pi \mu_{\mathrm{r}}\right)^{2} \mathrm{e}^{-2 \pi Z / k}
$$

Substituting this equation in (35) gives an extended version of Seaton's formula (3) which involves only the real part of the quantum defect:

$$
\cot \delta \approx\left[1-\mathrm{e}^{-2 \pi Z / k}-\left(\sin \pi \mu_{\mathrm{r}}\right)^{2} \mathrm{e}^{-4 \pi Z / k}\right] \cot \pi \mu_{\mathrm{r}} .
$$

We see that equation (38) differs from equation (3) of Seaton by a factor that includes the square of the exponent $\exp (-2 \pi Z / k)$. Thus equation (38) involves a second order correction to Seaton's threshold formula (2), whereas equation (3) involves a first order correction to (2). Of course, away from threshold, where $\mu_{\mathrm{i}}$ may not be small, the exact equations $(35,36)$ must be used to obtain $\delta$ as a function of $\mu$. Near threshold, we do not expect the extended $\delta-\mu$ relation to give an important improvement to predictions of phase shifts. For example, we compare in Table 1 our results with predictions of Seaton [36] and observe that for this value of kinetic energy, $k^{2} / 2$, our predictions are very close to that predicted by equation (3).

\section{QDT Green's function and eigenfunctions}

To illustrate the significance of the $\delta$ - $\mu$ relation (32) for theoretical analyses, we present here the QDT-analysis of the Green's function for the "generalized" Whittaker equation (7). We define the Green's function of the unperturbed Whittaker equation (having the asymptotic form of an outgoing spherical wave for $v=\mathrm{i} / k$ ) as is customary in physical applications (see, e.g., [37]):

$$
\hat{L}_{0}\left[G_{\lambda}^{0}\left(\nu ; z, z^{\prime}\right)\right]=-\nu \delta\left(z-z^{\prime}\right)
$$

Using the known Wronskian, $w\left[W, \mathcal{M}^{+}\right]$, of the functions (11) and employing the notation in (12), the solution of (39) 
can be written in a standard way as:

$$
\begin{aligned}
G_{\lambda}^{0}\left(\nu ; z, z^{\prime}\right) & =\nu \frac{\mathcal{M}^{+}\left(z_{<}\right) W\left(z_{>}\right)}{w\left[W, \mathcal{M}^{+}\right]} \\
& =\nu \Gamma(1+\lambda-Z \nu) \mathcal{M}^{+}\left(z_{<}\right) W_{Z \nu, \lambda+\frac{1}{2}}\left(z_{>}\right) .
\end{aligned}
$$

Here $z_{>}=(2 / v) \max \left(r, r^{\prime}\right)$, and $z_{<}=(2 / v) \min \left(r, r^{\prime}\right)$. It is a well-known fact that the Green's function contains all the necessary information on the Sturm-Liouville problem for the operator $\hat{\mathrm{L}}_{\mathrm{o}}$ and has an expansion in terms of the eigenfunctions of this problem (i.e., the spectral expansion). Namely, its poles determine the discrete spectrum of $\hat{\mathrm{L}}_{\mathrm{O}}$ (10) with an accumulation point at infinity. Above the threshold, equation (40) reduces with the help of (15) to the following form:

$$
\begin{aligned}
G_{\lambda}^{0}\left(\mathrm{i} / k ; r, r^{\prime}\right) & =\frac{2 \mathrm{i} \mathfrak{f}_{\lambda}\left(k, r_{<}\right)}{k}\left[\mathfrak{f}_{\lambda}\left(k, r_{>}\right)-\mathrm{ig}_{\lambda}\left(k, r_{>}\right)\right] \\
& \sim \frac{2 \sin \Delta_{\lambda}\left(r_{<}\right)}{k} \mathrm{e}^{\mathrm{i} \Delta_{\lambda}\left(r_{>}\right)} \quad \text { at } r, r^{\prime} \rightarrow \infty
\end{aligned}
$$

Obviously, the QDT Green's function $G_{\lambda}\left(v ; z, z^{\prime}\right)$ for the operator $\hat{\mathrm{L}}_{u}$ in the $r, r^{\prime}>r_{\mathrm{c}}$ domain (where $u(z)=0$ ) also satisfies equation (39), which is valid for the "unperturbed" Green's function $G^{o}\left(v ; z, z^{\prime}\right)$ at any $r, r^{\prime} \geq 0$. A general solution of (39) on the interval $r_{\mathrm{c}} \leq r, r^{\prime}<\infty$ can be expressed in terms of the Whittaker functions $(11,12)$ as follows:

$$
\begin{aligned}
G_{\lambda}\left(\nu ; z, z^{\prime}\right)= & \nu \Gamma(1+\lambda-Z \nu) \mathcal{M}^{+}\left(z_{<}\right) W_{Z \nu, \lambda+\frac{1}{2}}\left(z_{>}\right) \\
& +\mathrm{A} \nu W_{Z \nu, \lambda+\frac{1}{2}}(z) W_{Z \nu, \lambda+\frac{1}{2}}\left(z^{\prime}\right) \\
= & G_{\lambda}^{0}(\nu ; z, z)+\mathrm{A} \nu W_{Z \nu, \lambda+\frac{1}{2}}(z) W_{Z \nu, \lambda+\frac{1}{2}}\left(z^{\prime}\right) .
\end{aligned}
$$

The second term on the right-hand side of this equation is a particular solution of the homogeneous equation (39); for real $z, z^{\prime}$ this solution is regular over the interval considered. Since the Green's function must be regular at $r, r^{\prime} \rightarrow$ $\infty$, it must not contain the second particular solution, which is proportional to a product of two $\mathcal{M}^{+}$functions. The $r$-independent factor $\mathrm{A}$ depends on $v$ and $\lambda$ and is determined by the short-range core potential $u(z)$. In principle, it could be calculated by matching the infra-core value of $G_{\lambda}$ with its extra-core expression (43) at $r=r_{c}$. In particular, in the unperturbed case, (43) is valid for all $r$, and the boundedness condition at $r \rightarrow O$ leads to A $=0$. Since the Green's function in the $r, r^{\prime}<r_{\mathrm{c}}$ domain is unknown for $u(z) \neq 0$, we calculate $A$ for this case using the asymptotic form of (43) for imaginary $v$. Note that the asymptotic expression (42) remains valid in this case (taking into account the phase shift $\left.\delta_{\lambda}, c f .(28)\right)$ :

$$
\begin{aligned}
& G_{\lambda}\left(\mathrm{i} / k ; r, r^{\prime}\right) \sim \frac{2 \sin \left[\Delta_{\lambda}\left(r_{<}\right)+\delta_{\lambda}\right]}{k} \mathrm{e}^{\mathrm{i}\left[\Delta_{\lambda}\left(r_{>}\right)+\delta_{\lambda}\right]} \\
& \text { at } \quad r, r^{\prime} \rightarrow \infty
\end{aligned}
$$

So for imaginary $v$, we can express A in terms of the scattering phase. Taking into account equation (12) and substituting the asymptotic forms (16) into (43), and comparing the result with (44), we find

$$
\mathrm{A}=-\left[\mathrm{e}^{2 \mathrm{i} \delta_{\lambda}}-1\right] \exp \left[2 \mathrm{i} \sigma_{\lambda}+\mathrm{i} \pi(Z v-\lambda] .\right.
$$

If the phase $\delta_{\lambda}$ is known, equations $(43,45)$ determine the Green's function for the continuous spectrum (i.e., for imaginary $v$ ). Using (15) it can be written in a more compact form as

$$
\begin{array}{r}
G_{\lambda}\left(\mathrm{i} / k ; r, r^{\prime}\right)=\frac{2 \mathrm{ie}^{\mathrm{i} \delta_{\lambda}}}{k}\left[\mathfrak{f}_{\lambda}\left(k, r_{<}\right) \cos \delta_{\lambda}+\mathfrak{g}_{\lambda}\left(k, r_{<}\right) \sin \delta_{\lambda}\right] \\
\times\left[\mathfrak{f}_{\lambda}\left(k, r_{>}\right)-\mathrm{ig}_{\lambda}\left(k, r_{>}\right)\right]
\end{array}
$$

As is evident from (17), equation (46) leads immediately to the asymptotic expression (44). As in the unperturbed case, the imaginary part of the Green's function leads to the following expression for the continuum eigenfunction:

$$
f_{k \lambda}(r)=\sqrt{\frac{2}{\pi k}}\left(\mathfrak{f}_{\lambda}(k, r) \cos \delta_{\lambda}+\mathfrak{g}_{\lambda}(k, r) \sin \delta_{\lambda}\right) .
$$

For analytical continuation of expression (45) for A onto the real $v$ axis we use the previously obtained $\delta-\mu$ relation. Substituting (32) into (45) yields

$$
\mathrm{A}=\frac{\Gamma(1+\lambda-Z \nu)}{\Gamma(1+\lambda+Z \nu)} \frac{\sin \pi \mu_{\lambda}}{\sin \pi\left(\mu_{\lambda}+Z \nu-\lambda\right)} .
$$

So, for the discrete spectrum domain, the Green's function has the form given by (43) and (47).

A simple analysis shows that all of the above forms for the QDT Green's function coincide with the forms $(40,41)$ for the "unperturbed" Green's functions at $\mu_{\lambda} \rightarrow 0$. The QDT Green's function has poles determined by the condition (24), so that these poles correspond to the preassigned (e.g., experimental) energy levels. As in the unperturbed case, the residues at these poles,

$$
-\frac{1}{\nu^{3}} \operatorname{Res}_{\nu=\nu_{n \lambda}} G_{\lambda}\left(\nu ; z, z^{\prime}\right)=f_{n \lambda}(r) f_{n \lambda}\left(r^{\prime}\right),
$$

are equal to the product of the bound state eigenfunctions $f_{n \lambda}(r)$. Calculating the residue leads to

$$
f_{n \lambda}(r)=C_{n \lambda}^{-1 / 2}(-1)^{n} W_{Z \nu_{n \lambda}, \lambda+\frac{1}{2}}\left(\frac{2 r}{\nu_{n \lambda}}\right),
$$

where the normalization constant, $C_{n \lambda}$, given by

$$
\begin{aligned}
C_{n \lambda}=Z \nu_{n \lambda}^{2}\left(1+\left.\frac{1}{Z} \frac{\partial \mu_{\lambda}(\nu)}{\partial \nu}\right|_{\nu=\nu_{n \lambda}}\right) & \\
& \times \Gamma\left(Z \nu_{n \lambda}-\lambda\right) \Gamma\left(1+\lambda+Z \nu_{n \lambda}\right),
\end{aligned}
$$

is typical for the QDT wavefunctions [6]. By thus calculating the Green's function residues, this constant is obtained straightforwardly. 
Evidently, the QDT Green's function must not have the poles corresponding to the "unperturbed" eigenvalues (10). This fact is demonstrated most clearly if we rewrite $(43,47)$ to obtain,

$$
\begin{aligned}
& G_{\lambda}\left(\nu ; z, z^{\prime}\right)=-\frac{\pi \nu \cot \pi\left(\mu_{\lambda}+Z \nu-\lambda\right)}{\Gamma(Z \nu-\lambda) \Gamma(1+\lambda+Z \nu)} \\
& \quad \times W_{Z \nu, \lambda+\frac{1}{2}}(z) W_{Z \nu, \lambda+\frac{1}{2}}\left(z^{\prime}\right)+G_{\lambda}^{(\mathrm{s})}\left(\nu ; z, z^{\prime}\right),
\end{aligned}
$$

where

$$
\begin{aligned}
G_{\lambda}^{(\mathrm{s})}\left(\nu ; z, z^{\prime}\right)= & \nu \mathrm{e}^{\mathrm{i} \pi Z \nu} W_{Z \nu, \lambda+\frac{1}{2}}\left(z_{>}\right) W_{-Z \nu, \lambda+\frac{1}{2}}\left(-z_{<}\right) \\
& -\mathrm{i} \pi \nu \frac{W_{Z \nu, \lambda+\frac{1}{2}}(z) W_{Z \nu, \lambda+\frac{1}{2}}\left(z^{\prime}\right)}{\Gamma(Z \nu-\lambda) \Gamma(1+\lambda+Z \nu)}
\end{aligned}
$$

All the poles of $G_{\lambda}$ at positive $v$ are determined by the cotangent in the first, "resonant" term in (48), which is symmetric in $z, z^{\prime}$. Thus the "unperturbed" poles do not occur for $\mu_{\lambda} \neq 0$. The term $G_{\lambda}^{(s)}(v)$ in (48) does not contain any poles in the $v$-plane with the cut along the negative semiaxis, so it can be considered as the explicit form for one of the "smooth" Green's functions given in [15]. Note that this latter reference contains a discussion of different forms of "smooth" Green's functions, convenient in multichannel applications, in terms of different forms of expansions of both the bound and the continuum state wavefunctions. On the contrary, in equation (49) we have a closed form without any expansions.

It is important that the cotangent in the "resonance" term of (48) contains all of the dependence of $G_{\lambda}$ on $\mu_{\lambda}$. Thus, for $\mu_{\lambda}=0$, (48) gives an expression for the "unperturbed" Green's function in terms of its "resonant" and "smooth" parts. Note that the latter can also be written in another form having no complex quantities for real $v$ :

$$
\begin{aligned}
G_{\lambda}^{(\mathrm{s})}\left(v ; z, z^{\prime}\right) & =v \Gamma(1+\lambda-Z v) \\
& \times\left[\mathcal{M}^{+}\left(z_{<}\right)+\cos \pi(Z v-\lambda) \mathcal{W}^{+}\left(z_{<}\right)\right] W_{Z v, \lambda+1 / 2}\left(z_{>}\right) .
\end{aligned}
$$

Although $G_{\lambda}^{(\mathrm{s})}\left(v ; z, z^{\prime}\right)$ is a regular function of $v$, at $v=v^{0}{ }_{n \lambda}$ its representation (49) has ambiguous values of the type $\mathrm{o} \cdot \infty$. The numerical values of $G_{\lambda}^{(\mathrm{s})}$ at these points can be obtained by straightforward calculation of the limit as $v \rightarrow$ $v^{\mathrm{o}}$ or, alternatively, $G_{\lambda \lambda}^{(\mathrm{s})}\left(v^{\mathrm{o}}{ }_{n \lambda}\right)$ can be expressed in terms of the functions $\mathcal{W}^{ \pm}$, which are involved in (49) and are simplified at $v=v_{n \lambda}^{0}$ and $z=z_{n}=2 \mathrm{r} / v_{n \lambda}^{0}$ as follows:

\section{[see equation (50-A) below]}

where $L_{n}^{\alpha}$ is the generalized Laguerre polynomial and $U$ is the confluent hypergeometric function of the second kind [31].

A "smooth-pole" decomposition of the "non-perturbed" Coulomb Green's function $G_{\lambda}^{0}$, somewhat similar to (48), was stated in [19]. We correct a misprint occurring in that work and re-write the result in terms of the functions used in the present paper:

\section{[see equation (51) below]}

where $\mathcal{Y}_{\beta}(z)$ is defined in equation (A.2) of Appendix A. Since it is defined to within an arbitrary regular function of $v$, this is an alternative definition to that stated above (i.e., Eq. (48) with $\mu_{\lambda}=0$ ). The difference between these definitions is that the result in equation (48) is an analytical function of $v$, while the result in equation (51) is not. More specifically, the result in (51) is analytic only for noninteger $2 \lambda$; otherwise, another "smooth-pole" decomposition should be used.

It should be noted that the QDT Green's function for the discrete spectrum domain can be obtained in a form similar to that in equation (40) if we take $f_{\lambda}^{\text {reg }}$ as the regular solution and $W$ as the irregular one:

$$
G_{\lambda}\left(\nu ; z, z^{\prime}\right)=\nu \frac{f_{\lambda}^{\mathrm{reg}}\left(z_{<}\right) W_{Z \nu, \lambda+\frac{1}{2}}\left(z_{>}\right)}{w\left[W_{Z \nu, \lambda+\frac{1}{2}}, f_{\lambda}^{\text {reg }}\right]} .
$$

Using (27) and calculating the Wronskian, $w$, easily yields the same result as in $(43,47)$ for real $v$. In this way the QDT Green's function was constructed in [15]. But unlike equation (40), which contains well-known functions and has the same form for all $v$, the $\delta-\mu$ relation (32) is needed for the direct continuation of $G_{\lambda}(v)$ in equation (52) for real $v$ to

$$
\begin{aligned}
G_{\lambda}^{(\mathrm{s})}\left(\nu_{n \lambda}^{0} ; z_{n}, z_{n}^{\prime}\right)= & \nu_{n \lambda}^{0} n !\left(z_{n} z_{n}^{\prime}\right)^{\lambda+1} \mathrm{e}^{-\left(z_{n}+z_{n}^{\prime}\right) / 2} L_{n}^{2 \lambda+1}\left(z_{>, n}\right) \\
& \times\left\{-\frac{\mathrm{i} \pi}{\Gamma(n+2 \lambda+2)} L_{n}^{2 \lambda+1}\left(z_{<, n}\right)+\mathrm{e}^{z<, n} \mathrm{e}^{2 \pi \mathrm{i} \lambda} U\left(n+2 \lambda+2,2 \lambda+2 ;-z_{<, n}\right)\right\},
\end{aligned}
$$

$$
\begin{aligned}
G_{\lambda}^{0}\left(\nu ; z, z^{\prime}\right)= & -\pi \nu \frac{\Gamma(1+\lambda+Z \nu)}{\Gamma(-\lambda+Z \nu)} \mathcal{M}^{+}(z) \mathcal{M}^{+}\left(z^{\prime}\right) \cot \pi(Z \nu-\lambda) \\
& -\pi \nu \frac{\mathcal{M}^{+}\left(z_{<}\right)}{\sin 2 \pi \lambda}\left[\cos 2 \pi \lambda \frac{\Gamma(1+\lambda+Z \nu)}{\Gamma(-\lambda+Z \nu)} \mathcal{M}_{Z \nu, \lambda+\frac{1}{2}}\left(z_{>}\right)+\mathcal{M}_{Z \nu,-\lambda-\frac{1}{2}}\left(z_{>}\right)\right] \\
= & -\pi \mathcal{A}(\nu, \lambda) \mathrm{f}_{\lambda}(z) \mathrm{f}_{\lambda}\left(z^{\prime}\right) \cot \pi(Z \nu-\lambda)+\pi \mathrm{f}_{\lambda}\left(z_{<}\right) \mathcal{Y}_{\beta}\left(\nu, \lambda ; z_{>}\right)
\end{aligned}
$$


the continuum domain. The same arguments are valid for the continuation of equations $(43,45,46)$ to the real $v$ domain. The situations considered here are simple examples of the use of relations (32): they allow one to avoid routine calculations. Indeed, otherwise one needs to obtain separate expressions for the solutions with positive and negative energies and to trace accurately the analytic continuation between them.

An important role in the Green's function approach is played by integral representations. One such representation was given for the "unperturbed" Coulomb Green's function (40) in [37]. The result contains the integral of the Bessel function for an imaginary argument, $I_{2 \lambda+1}(x)$. Using the known integral representation for the product of two Whittaker functions $\mathcal{W}^{+}$[38], we present here an analogous integral representation for the "smooth" Green's function:

\section{[see equation (53) below, ]}

where $K_{2 \lambda+1}(x)$ is the McDonald function. Using the properties of Bessel and McDonald functions, it can be shown that the "smooth" Green's function (53) has no peculiarities at the Coulomb pole points $v=v_{n \lambda}^{0}=(n+\lambda+1) / Z$.

\section{Summary and conclusions}

In this paper we have presented a theoretical analysis of the relationship between the quantum defect and the phase shift for a single electron described by an equation having the general form of the Whittaker equation including a short-range potential. The relationship obtained requires that $\mu$ becomes complex for energies above threshold, which is complementary to the known fact that the phase shift becomes complex below threshold. For the case of integer $\lambda$ $=l$, this general relationship (32) may be regarded as extending Seaton's relation between $\delta_{l}$ and $\mu_{l}$. In our view, the most important application for the general relation (32) for arbitrary $\lambda$ is in analytical work; in particular, to construct the single-channel Green's function in a completely general form ( $c f$. Equations $(43,45,47))$ in terms of the phase shift for energies above threshold and the quantum defect for energies below threshold. We have presented compact analytic representations for the Green's function in terms of its resonant and smooth parts (see Equations (48-51)), and have also given an integral representation (53) for the smooth part. All of our general results have been related to earlier, more specialized results (where they exist). We ex- pect the general forms of the Green's functions we present here, which are obtained using the relationships presented in (32), to have practical applications in theoretical work. In particular we expect these Green's functions will aid computations of high-order perturbation treatments of multiphoton processes for electrons in a screened Coulomb or Coulomb plus dipole potential. Finally, while we have dealt in this work only with the single-channel QDT, the generalization of the QDT-approach to multichannel (e.g., collisional or molecular) problems is well-known and details can be found in [6,39-41]. Results presented here are also useful for multichannel applications, particularly for the construction of multichannel QDT-Green's functions. However, we have deferred this extension to a subsequent work.

N. L. Manakov gratefully acknowledges the hospitality of the University of Nebraska-Lincoln where a part of this work was carried out during his visit to the Department of Physics and Astronomy. A. F. Starace thanks Paul Koosis for useful comments. This research was supported in part by the Russian Foundation for Basic Research (Grants 98-02-16111 and 97-02-18035), by the Grant Program "Universities of Russia," and by the US National Science Foundation through Grant No. PHY-9722110.

\section{Appendix A: Alternative derivation of the $\delta-\mu$ relation in terms of $\mathcal{Y}_{\alpha}$ and $\mathcal{Y}_{\beta}$ basis functions}

Besides the functions mentioned in Section 2.1, another basis pair is widely used in QDT. This pair is denoted (to within a constant factor) as $f, \eta$ in [6], as $y_{1,3}$ in [7], and as $\mathbf{y}_{\alpha, \beta}$ in [24]. These base functions are analytic in $v$ near threshold for $2 \lambda \neq 2 l$. In the present paper they are denoted as $\mathcal{Y}_{\alpha, \beta}(v, \lambda ; z)$, which we express in terms of the $\left(\mathcal{M}^{+}, \mathcal{W}^{+}\right)$, $\mathcal{W}^{ \pm},\left(\mathfrak{f}_{\lambda}, \mathfrak{f}_{-\lambda-1}\right)$, and $\left(\mathfrak{f}_{\lambda}, \mathfrak{g}_{\lambda}\right)$ base pairs as follows:

$$
\begin{aligned}
\mathcal{Y}_{\alpha} & =\mathrm{f}_{\lambda}=\nu^{\lambda+1} \mathcal{M}^{+} \leftrightarrow \frac{2 \mathfrak{f}_{\lambda} k^{-(\lambda+1)} \mathrm{e}^{-\frac{\pi Z}{2 k}}}{\left|\Gamma\left(1+\lambda+\mathrm{i} \frac{Z}{k}\right)\right|} \\
\mathcal{Y}_{\beta} & =-\csc 2 \pi \lambda\left[\mathrm{f}_{\lambda} \cos 2 \pi \lambda+\mathcal{A}(\nu, \lambda) \mathrm{f}_{-\lambda-1}\right] \\
& =\nu^{\lambda+1}\left[\mathcal{M}^{+} \cot \pi(Z \nu-\lambda)+\mathcal{W}^{+} \csc \pi(Z \nu-\lambda)\right] \\
& =\nu^{\lambda+1} \mathrm{e}^{\mathrm{i} \pi(Z \nu-\lambda)}\left[\mathrm{e}^{\mathrm{i} \pi \lambda} \mathcal{W}^{-} \cot \pi(Z \nu-\lambda)-\mathrm{i} \mathcal{W}^{+}\right] \\
& \leftrightarrow \frac{2 k^{-(\lambda+1)} \mathrm{e}^{-\frac{\pi Z}{2 k}}}{\left|\Gamma\left(1+\lambda+\mathrm{i} \frac{Z}{k}\right)\right|} \frac{\mathfrak{g}_{\lambda}-\mathrm{if}_{\lambda} \mathrm{e}^{-\frac{2 \pi Z}{k}-\mathrm{i} 2 \pi \lambda}}{1-\mathrm{e}^{-\frac{2 \pi Z}{k}-\mathrm{i} 2 \pi \lambda}}
\end{aligned}
$$

Using this base pair, we provide here another way to deduce the $\delta-\mu$ relation (32). Assuming for the moment that

$$
\begin{aligned}
G_{\lambda}^{(\mathrm{s})}\left(\nu ; z, z^{\prime}\right)= & \nu \sqrt{z z^{\prime}} \int_{0}^{1} \mathrm{~d} t \frac{t^{-Z \nu-\frac{1}{2}}}{1-t} \exp \left[-\frac{1}{2}\left(z+z^{\prime}\right) \frac{1+t}{1-t}\right] \\
& \times\left[I_{2 \lambda+1}\left(\frac{\sqrt{z z^{\prime} t}}{1-t}\right)-\frac{2}{\pi} \sin \pi(Z \nu+\lambda) \cos \pi(Z \nu-\lambda) K_{2 \lambda+1}\left(\frac{\sqrt{z z^{\prime} t}}{1-t}\right)\right]
\end{aligned}
$$


$\mu$ is some parameter, we express the regular solution $f_{\lambda}^{\text {reg }}$ (21) in terms of $\mathcal{Y}_{\alpha}$ and $\mathcal{Y}_{\beta}$ :

$$
f_{\lambda}^{\text {reg }}=N\left(\cot \pi \mu \mathcal{Y}_{\alpha}+\mathcal{Y}_{\beta}\right)
$$

Thus, instead of the coefficient $\beta(v)$ in (21), we parameterize the regular solution $f_{\lambda}^{\text {reg }}$ by another auxiliary function, cot $\pi \mu(v)$. For integer $2 \lambda=2 l$, the $\mu$ parameter in (A.5) is the quantum defect [6]. For arbitrary $\lambda$, substituting (A.1, A.2) into (A.5) and comparing the result with (21) we find that $N$ $=[\cot \pi \mu-\cot 2 \pi \lambda]^{-1}$. It is evident from (27) that the exponentially increasing part of (A.5) vanishes only for that $v$ which satisfies the bound state condition (24). This fact shows that $\mu$ in (A.5) is indeed the quantum defect also for non-integer $\lambda$. On the other hand, in the above-threshold domain, the regular solution (A.5) should have the asymptotic form (28). Substituting (A.1, A.4) into (A.5) we may express $f_{\lambda}^{\text {reg }}$ in terms of the $\left(\mathfrak{f}_{\lambda}, \mathfrak{g}_{\lambda}\right)$-pair. Using the asymptotic relations (17) for $\mathfrak{f}_{\lambda}, \mathfrak{g}_{\lambda}$ and comparing the result with (28), we obtain our basic $\delta-\mu$ relations (32) once again.

As noted above, $\mathcal{Y}_{\beta}$ is not an analytic function of $v$ for integer values of $2 \lambda$. In this case an analytic solution, $\mathcal{Y}_{\gamma}$, can be constructed using the procedure proposed in [6]:

$$
\mathcal{Y}_{\gamma}(z)=\mathcal{Y}_{\beta}(z)-\mathrm{G} \mathcal{Y}_{\alpha}, \quad \mathrm{G}(\nu, l)=\left.\frac{1}{2 \pi} \frac{\mathrm{d} \mathcal{A}(\nu, \lambda)}{\mathrm{d} \lambda}\right|_{\lambda=l} .
$$

Note that Fano and coworkers refer to $\mathcal{G}=\operatorname{Re} G$ as one of the six QDT parameters [15]. Since, so far as we know, the explicit form for $\mathcal{Y}_{\gamma}(z)$ has not been presented elsewhere, we provide it here:

\section{[see equation (A.6) below]}

where $\psi(z)$ is the logarithmic derivative of $\Gamma(z)$. It is easy to see that $\mathcal{Y}_{\gamma}$ has only a finite number of poles at $v=-l, \ldots$ , l. It is thus analytic near threshold, i.e., for large $v$. These considerations apply as well for half-integer $\lambda$.

\section{Appendix B: Application to special cases of the Whittaker equation}

In this appendix we indicate the physical meaning of the parameters $Z, \lambda, v, \mu_{\lambda}$ for some concrete problems for which particular, appropriate Green's functions and $\delta-\mu$ relations can be obtained as limiting cases of the general results presented above.
Non relativistic electron in a hydrogen-like atom or ion

The radial Schrödinger equation for an electron in a Coulomb plus short range potential reduces to equation (7), where

$-\lambda=l$ is the (integer) orbital angular momentum quantum number;

$-Z$ is the atomic core (or ion) charge;

$-v$ is related to the energy $\varepsilon$ (in atomic units) as $v=1 /(-$ $2 \varepsilon)^{1 / 2}$;

- energy levels: $\varepsilon_{n l}=-Z^{2} /\left(2\left(n+l+1-\mu_{n l}\right)^{2}\right)$

The QDT Green's function for the non-relativistic Coulomb problem was constructed for the first time in [11], where it had a form similar to that given in (43-47), but with integer $\lambda=l$.

\section{Relativistic electron in a hydrogen-like atom or ion}

In the domain $r>r_{c}$ the squared radial Dirac equation for an electron in a Coulomb plus short range potential reduces to equation (7) with $u(z)=0$, where

$-v$ is related to the energy $E={ }^{-} m_{e} c^{2}$ by $v=\alpha /\left(1-\frac{-2}{\varepsilon}\right)^{1 / 2}$. Here $m_{e}$ is the electron mass, $c$ is the speed of light, and $\alpha$ is the fine structure constant;

$-Z$ must be replaced by ${ }^{-} \mathcal{Z}$, where $\mathcal{Z}$ is the core charge of the atom or ion;

$-\lambda$ is the relativistic parameter connected with the total $(j)$ and orbital $(l)$ angular momentum of the electron:

$\lambda=\left[(j+1 / 2)^{2}-(\alpha \mathcal{Z})^{2}\right]^{1 / 2}+l-j-1 / 2$

- energy levels:

$$
E_{n \lambda}=m_{\mathrm{e}} c^{2}\left[1+\left(\frac{\alpha \mathcal{Z}}{n+\lambda+1-\mu_{n \lambda}}\right)^{2}\right]^{-\frac{1}{2}} .
$$

Here $n$ coincides with the radial quantum number $n_{\mathrm{r}}$ for $l=j-1 / 2$ and $n_{\mathrm{r}}=n+1$ at $l=j+1 / 2$.

The full three-dimensional QDT Green's function for the relativistic Coulomb problem has been presented in [28].

\section{Rydberg electron in a polar molecule}

As was shown in [42], the Rydberg states of polar molecules can be described by a Schrödinger equation for an electron in a point dipole plus Coulomb potential. The angular

$$
\begin{aligned}
\mathcal{Y}_{\gamma}(z)= & \mathcal{Y}_{\beta}(z)+\frac{1}{2 \pi}[\psi(1+l+Z \nu)+\psi(Z \nu-l)-2 \ln \nu] \mathcal{Y}_{\alpha}(z) \\
= & {\left[\ln 2 r+\cot (Z \nu-l)+\frac{1}{2 \pi} \psi(1+l+Z \nu)+\frac{1}{2 \pi} \psi(Z \nu-l)\right] \mathcal{Y}_{\alpha}(z) } \\
& +\frac{(z \nu)^{l+1} \mathrm{e}^{-z / 2}}{\pi \Gamma(1+l-Z \nu)} \sum_{k=0}^{\infty} \frac{\Gamma(1+l-Z \nu+k)}{(2 l+k+1) !}[\psi(1+l-Z \nu+k)+\psi(2 l+2+k)+\psi(1+k)] \frac{z^{k}}{k !} \\
& -\nu \frac{(\nu / z)^{l} \mathrm{e}^{-z / 2}}{\pi \Gamma(1+l-Z \nu)} \sum_{k=0}^{2 l} \Gamma(-l-Z \nu+k)(2 l-k) ! \frac{(-1)^{k} z^{k}}{k !}
\end{aligned}
$$


variables can be separated for this problem, and the radial Schrödinger equation reduces to equation (7), where

$-\lambda=\lambda(l, m, d)$ depends on the projection $m$ of the electron orbital momentum $l$ onto the molecular axis as well as on the permanent molecular dipole moment $d$. Being a non-integer analogue of the atomic electron orbital quantum number, $\lambda$ values are enumerated by integer $l$ so that $\lambda \rightarrow l$ as $d \rightarrow 0$;

$-Z$ is the molecular core (or ion) charge;

$-v$ is related to the energy $\varepsilon$ as $v=1 /(-2 \mathrm{e})^{1 / 2}$;

- energy levels: $\varepsilon_{n \lambda}=-\frac{Z^{2}}{2\left[n+\lambda(l, m, d)+1-\mu_{n \lambda}\right]^{2}}$.

The QDT for the radial Schrödinger equation with a point dipole plus Coulomb potential was discussed in $[16,33]$, and has been used in atomic calculations (see, e.g., [43]). The three-dimensional QDT-Green's function for a polar Rydberg molecule was constructed in [29].

\section{Appendix C: On the relation of the present results to the generalized form of QDT}

In this appendix we discuss in more detail (than in the Introduction) the relation of our results to the general form of QDT developed by Fano and coworkers. In particular, we consider the main issue discussed in the Appendix of reference [15] concerning the asymptotic form of the regular Coulomb solution for negative energies. Note that some functions mentioned above in Section 2.1 are referred to in references [15-17,33] using another notation, e.g., they employ $f^{ \pm}$and $f^{\circ}$, which are defined by

$$
\begin{array}{r}
f^{ \pm}=\left( \pm \frac{\nu}{2}\right)^{ \pm Z \nu} W_{ \pm Z \nu, \lambda+\frac{1}{2}}( \pm z) \rightarrow r^{ \pm Z \nu} \mathrm{e}^{\mp r / \nu} \\
\text { at } r \rightarrow \infty, \\
f^{0}=\frac{\Gamma(2 \lambda+2)}{2^{\lambda+1}} \mathrm{f}_{\lambda}=\left(\frac{\nu}{2}\right)^{\lambda+1} \Gamma(2 \lambda+2) \mathcal{M}^{+}(z) .
\end{array}
$$

The regular function at the origin, $f^{\circ}$, in the above-cited works is given separate representations above and below the threshold:

$$
\begin{aligned}
f^{0} & \left.=\frac{\mathrm{i}}{\sqrt{2 \pi k B}}\left(f^{-} \mathrm{e}^{-\mathrm{i} \eta}-f^{+} \mathrm{e}^{\mathrm{i} \eta}\right), \quad \varepsilon>0, \quad \text { (C. } 3\right) \\
& \left.=\frac{1}{\sqrt{\pi k A}}\left(D^{-1} \sin \beta f^{-}-D \cos \beta f^{+}\right), \quad \varepsilon<0 \text {. (C. } 4\right)
\end{aligned}
$$

The parameters $\mathrm{A}, B, D, \eta$, and $\beta$ involved in the above equations are the (real) QDT parameters introduced in references $[15,16]$. (The last, sixth QDT parameter, $\mathcal{G}=\operatorname{Re} \mathrm{G}$, was noted in Appendix A.) Using the standard definition (12) for $\mathcal{M}^{+}(z)$ in terms of $\mathcal{W}^{ \pm}(z)$ functions, the regular Coulomb solution $f^{\circ}$ for $\epsilon<0$ may be presented in the same form as (C.4), with the same parameters $\eta$ and $\beta$, i.e.,

$$
\eta=\frac{Z}{k} \ln 2 k-\frac{\pi \lambda}{2}-\sigma_{\lambda} \quad \text { and } \quad \beta=\pi(Z \nu-\lambda),
$$

but with complex parameters $A$ and $D$. The expressions one finds in this way for $A$ and $D$ are:

$$
\begin{aligned}
D_{\mathrm{c}}^{2} & =\mathrm{e}^{\mathrm{i} \beta}\left(\frac{2}{\nu}\right)^{2 Z \nu} \frac{\pi \sec \beta}{\Gamma(Z \nu-\lambda) \Gamma(1+\lambda+Z \nu)}, \\
A_{\mathrm{c}} & =\mathrm{e}^{-\mathrm{i} \beta} \cos \beta \frac{2^{2 \lambda+2}}{\Gamma^{2}(2 \lambda+2)} \mathcal{A}(\nu, \lambda),
\end{aligned}
$$

where we have distinguished the complex parameters by a subscript c. The (real) expressions for $D$ and $A$ used by Fano and coworkers in equation (C.4) follow from (C.6) upon substituting

$$
\exp (\mathrm{i} \beta) \rightarrow \cos \beta, \quad \exp (-\mathrm{i} \beta) \rightarrow \sec \beta .
$$

i.e., $D^{2}=\operatorname{Re} D_{\mathrm{c}}^{2}$ and $A^{-1}=\operatorname{Re}\left(A_{\mathrm{c}}^{-1}\right)$. The origin of this difference-and this is the key (mathematical) difference between the unified development of QDT and previous studies in QDT-is that in the above cited works the authors used another asymptotic expression for the confluent hypergeometric function $F(a, c, z)$-and therefore for $M_{k, m}(z)=\exp$ $(-z / 2) z^{m+1 / 2} F(m-k+1 / 2,2 m+1, z)$-from that given in standard textbooks, which the authors of reference [15] denote the "apparent large- $z$ form" (see the Appendix in Ref. [15]). In fact, in reference [15] the substitution $\exp (\mathrm{i} \beta) \rightarrow$ $\cos \beta$ is made in the complex factor before the term with decreasing exponent in the standard asymptotic form of $F(1+$ $\lambda-v, 2 \lambda+1, z)$ for $z=2 r / v>0$.

In our analysis we have not used the alternate asymptotic form of the regular solution employed in [15] since in our derivation in Section 2.2 we do not need in fact a detailed form of the factor before the decreasing term in the asymptotic form of $f_{\lambda}^{\text {reg }}(v ; z \rightarrow \infty)$ for real $v$. To establish the parameter $\beta$ in equation (21) for bound state energies we employ only the fact that the term with the increasing exponent must vanish for $v=v_{n}$ in order to derive equation (23). The standard asymptotic form of $F(a, c, z)\left(\right.$ or $\left.\mathcal{M}^{+}\right)$ follows from the well-known relation (12) between $M$ and $W$ functions and asymptotic expansions (16) for $W$ functions, and does not seem to require another derivation. The complexity of the manifestly real regular solution for $\epsilon<0$ at large $r$-which is postulated in the Appendix of [15] to be an inaccuracy of the standard asymptotic form for $F(a$, $c, z)$-is only apparent, since for negative energies the term with decreasing exponent should be dropped in the asymptotic expansion of $F(a, \mathrm{c}, z)$ in comparison with the term with increasing exponent. For negative energy not equal to a bound state energy, the exponentially increasing terms in the asymptotic expansion of $F(a, c, z)$ are the only ones which matter, and the fact that the coefficient of the exponentially decreasing term is complex is of no consequence. For bound state energies, of course, the coefficient of the exponentially increasing term vanishes and the coefficient of the decreasing exponential (which must now be kept) is real. Owing to the singularity of a Coulomb-like potential, there may not exist for the regular solution with $\epsilon$ $<0$ an accurate representation at large $r$ in the form of a sum of two real terms with increasing and decreasing expo- 
nents $\exp ( \pm r / v)$ (with the exception, obviously, of the case $\epsilon=\epsilon_{n}$ when there remains only one real term with decreasing exponent). In reference [15], the authors presented more physical rather than mathematical arguments to justify their corrected large-z asymptotics. Although they write that "It [i.e., their prescribed asymptotic form] could presumably be justified in greater detail ..." by considering the representation of $F(a, \mathrm{c}, z)$ for $\epsilon<O$ as a contour integral along a double loop in the complex plane, they do not justify it in this way.

We regard the large- $r$ representation suggested in [15] for the regular solution as a physically-based prescription for introducing the Jost formalism into the QDT analysis for singular potentials. The results of such analysis demonstrate the utility of this suggested approach for applications. Taking into account the discussion in the Introduction and the above comments, we conclude that there is no contradiction between the generalized form of QDT and the derivations in this paper since nonoverlapping matters are considered. We agree with the authors of reference [16] (cf. p. $2443)$ that the analysis of $\delta_{\lambda}(v)$ " “... is regarded as a problem of core dynamics to be pursued separately." Nevertheless, this dynamics introduces energy dependences of both functions, $\mu(v)$ and $\delta(v)$, which may not be the same. Moreover, a general relation between these two functions may exist, which is independent of the details of the short-range interactions, as demonstrated by (32).

\section{References}

1. D. Bates, A. Damgaard, Phil. Trans. Roy. Soc. A 242, 101 (1949).

2. M. J. Seaton, Compt. Rend. 240, 1317 (1955).

3. M. J. Seaton, Mont. Not. Roy. Astr. Soc. 118, 504 (1958).

4. F. S. Ham, in Solid State Physics, edited by F. Seitz, D. Turnbull (Academic Press, New York, 1955), Vol. 1.

5. A. Burgess, M. J. Seaton, Mont. Not. Roy. Astr. Soc. 120, 121 (1960).

6. M. J. Seaton, Rep. Prog. Phys. 7o, 167 (1982).

7. M. J. Seaton, Proc. Phys. Soc. 88, 801 (1966).

8. B. L. Moiseiwitsch, Proc. Phys. Soc. Lond. 79, 1166 (1962).

9. G. E. Norman, Opt. Spektrosk. 12, 333 (1962) [Opt. Spectrosc. (USSR) 12, 183 (1962)].

10. L. D. Landau, Ya. A. Smorodinskii, J. Phys. USSR 8, 154 (1944).

11. B. A. Zon, N. L. Manakov, L. P. Rapoport, Doklady Akad. Nauk USSR 188, 560 (1969) [Sov. Phys.-Dokl. 14, 904 (1970)].

12. V. A. Davidkin, B. A. Zon, N. L. Manakov, L. P. Rapoport, Zh. Eksp. Teor. Fiz. 6o, 124 (1971) [JETP 33, 70 (1971)].

13. N. L. Manakov, V. D. Ovsiannikov, L. P. Rapoport, Phys. Rep. 141, 319 (1986).
14. I. Fabrikant, D. Khrebtukov, Phys. Rev. A 54, 2906 (1996).

15. C. Greene, U. Fano, G. Strinati, Phys. Rev. A 19, 1485 (1979).

16. C. Greene, A. R. P. Rau, U. Fano, Phys. Rev. A 26, 2441 (1982).

17. A. R. P. Rau, Phys. Rev. A 38, 2255 (1988).

18. U. Fano, Phys. Rev. A 17, 93 (1978).

19. L. P. Presnyakov, A. M. Urnov, Zh. Eksp. Theor. Phys. 68, 61 (1975) [JETP 41, 31 (1975)].

20. T. L. Goforth, D. K. Watson, Phys. Rev. A 46, 1239 (1992).

21. W. A. Henie, H. Ritsch, P. Zoller, Phys. Rev. A 36, 683 (1987).

22. J. A. Stephens, V. McKoy, J. Chem. Phys. 97, 8060 (1992).

23. V. A. Zilitis, Opt. Spektrosk. 43, 1017 (1977) [Opt. Spectrosc. (USSR) 43, 603 (1977)].

24. W. R. Johnson, K. T. Cheng, J. Phys. B 12, 863 (1979).

25. V. A. Zilitis, Opt. Spektrosk. 5o, 419 (1981) [Opt. Spectrosc. (USSR) 50, 227 (1981)].

26. I. B. Goldberg, R. H. Pratt, J. Math. Phys. 28, 1352 (1987).

27. J. J. Chang, Phys. Rev. A 48, 1769 (1993).

28. S. A. Zapryagaev, N. L. Manakov, A. V. Mogilev, Izv. Akad. Nauk SSSR: Ser. Fiz. 5o, 1367 (1986) [Bull. Acad. Sci. USSR: Phys. Ser. 5o, 120 (1986)].

29. V. E. Chernov, B. A. Zon, J. Phys. B 29, 4161 (1996).

30. P. M. Morse, H. Feshbach, Methods of Theoretical Physics (McGraw-Hill, New York, 1953), Vol. I, pp. 348-349.

31. L. J. Slater, Confluent Hypergeometric Functions (Cambridge University Press, Cambridge, England, 1960).

32. E. T. Whittaker, G. N. Watson, Modern Analysis (Cambridge University Press, Cambridge, England, 1973).

33. U. Fano, A. R. P. Rau, Atomic Collisions and Spectra (Academic Press, New York, 1986).

34. M. Abramowitz, I. A. Stegun, Handbook of Mathematical Functions (Dover, New York, 1965), p. 256.

35. V. De Alfaro, T. Regge, Potential scattering (North-Holland, Amsterdam, 1965).

36. M. J. Seaton, Proc. Phys. Soc. A 70, 620 (1957).

37. L. H. Hostler, J. Math. Phys. 5, 591 (1964).

38. I. S. Gradshtein, I. M. Ryzhik, Tables of Integrals, Series and Products (Academic Press, New York, 1967), Eq. 6.669(3).

39. U. Fano, Phys. Rev. A 2, 353 (1970).

40. C. M. Lee, Phys. Rev. A 10, 584 (1974).

41. M. Aymar, C. H. Greene, E. Luc-Koenig, Rev. Mod. Phys. 68, 1015 (1996).

42. B. A. Zon, Zh. Eksp. Theor. Phys. 102, 36 (1992) [JETP 75, 19 (1992)].

43. N. Y. Du, A. F. Starace, N. A. Cherepkov, Phys. Rev. A 48, 4213 (1993). 